\title{
Sources of total, non-milk extrinsic, and intrinsic and milk sugars in the diets of older adults living in sheltered accommodation
}

\author{
Jane Bradbury ${ }^{1}$, Charlotte E. Mulvaney ${ }^{1}$, Ashley J. Adamson ${ }^{2}$, Chris J. Seal ${ }^{3}$, John C. Mathers ${ }^{2}$ \\ and Paula J. Moynihan ${ }^{1 *}$ \\ ${ }^{1}$ Human Nutrition Research Centre, School of Dental Sciences, Newcastle University, Newcastle upon Tyne, NE1 7RU, UK \\ ${ }^{2}$ School of Clinical Medical Sciences, Newcastle University, Newcastle upon Tyne NE1 7RU, UK \\ ${ }^{3}$ School of Agriculture, Food and Rural Development, Newcastle University, Newcastle upon Tyne NE1 7RU, UK
}

(Received 28 February 2007 - Revised 4 June 2007 - Accepted 10 July 2007)

The WHO recommends limiting non-milk extrinsic sugars (NMES) consumption to $\leq 10 \%$ energy to reduce the risk of unhealthy weight gain and dental caries, and to restrict frequency of intake to $\leq 4$ times/d to reduce risk of dental caries. Older adults, especially those from lowincome backgrounds, are at increased risk of dental caries, yet there is little information on sugars intake (frequency of intake and food sources) in this age group. The aim of this report is to present baseline data from a community-based dietary intervention study of older adults from socially deprived areas of North East England, on the quantity and sources of total sugars, NMES, and intrinsic and milk sugars, and on frequency of NMES intake. Dietary intake was assessed using two 3-d estimated food diaries, completed by 201 participants (170 female, thirty-one male) aged 65-85 years (mean 76.7 (SD 5.5) years) recruited from sheltered housing schemes. Total sugars represented $19.6 \%$, NMES $9.3 \%$, and intrinsic and milk sugars $10.3 \%$ of daily energy intake. Eighty-one (40.3\%) exceeded the NMES intake recommendation. Mean frequency of NMES intake was 3.4 times/d. The fifty-three participants $(26.4 \%)$ who exceeded the frequency recommendation $(\leq 4$ times/d) obtained a significantly greater percentage of energy from NMES compared with those participants who met the recommendation. The food groups 'biscuits and cakes' (18.9\%), 'soft drinks' (13.1\%) and 'table sugar' (11.1\%) made the greatest contributions to intakes of NMES. Interventions to reduce NMES intake should focus on limiting quantity and frequency of intake of these food groups.

Non-milk extrinsic sugars: Sugars: Dietary intakes: Older adults

In the report Dietary Sugars and Human Disease ${ }^{(1)}$, sugars were classified into intrinsic and extrinsic sugars. Extrinsic sugars were defined as sugars not located within the cellular structure of food, and were further divided into milk sugars (lactose, naturally present in milk and dairy foods) and nonmilk extrinsic sugars (NMES). The term NMES, generally only adopted in the UK, is synonymous with the term 'free sugars': 'all monosaccharides and disaccharides added to foods by the manufacturer, cook or consumer, plus sugars naturally present in honey, syrups and fruit juices' used by $\mathrm{WHO}^{(2)}$. WHO recommend that to reduce the risk of dental caries (tooth decay), free sugars should not exceed $10 \%$ of total daily energy intake ${ }^{(3)}$, and the UK dietary reference value for NMES is also $\leq 10 \%$ of total daily energy intake, or $\leq 11 \%$ of food energy ${ }^{(4)}$. Frequency of NMES intake is also associated with the development of caries ${ }^{(3,5)}$; it is recommended to limit consumption to $\leq 4$ times/d to reduce risk $^{(3,6)}$.
Loss of teeth, once considered an inevitable consequence of ageing, has slowed dramatically with increased preventive measures such as exposure to fluoride and improved oral hygiene ${ }^{(7)}$. Increased tooth retention in older adults has led to increased prevalence of dental caries, with those from socially deprived backgrounds most at risk ${ }^{(8)}$. Caries of the root surfaces is a particular problem for older adults: the root surface becomes exposed due to gingival recession as a result of episodes of periodontal disease, and risk factors such as a dry mouth, or wearing partial dentures, are more common in older people ${ }^{(9)}$. Intake of NMES is strongly related to root caries in older adults ${ }^{(9,10)}$.

There are few published data on NMES intakes of older adults worldwide, particularly dietary sources of NMES and frequency of intake. In the USA the 1994-1996 Continuing Survey of Food Intakes by Individuals (CSFII), 'added sugars' contributed $12.5 \%$ of daily energy intake $(46 \mathrm{~g} / \mathrm{d})$ for women aged $\geq 60$ years and $11.6 \%$ of daily energy intake $(59 \mathrm{~g} / \mathrm{d})$ for

Abbreviations: NMES, non-milk extrinsic sugars; PAL, physical activity level; CSFII, Continuing Survey of Food Intakes by Individuals; HNRC, Human Nutrition Research Centre; NDNS, National Diet and Nutrition Survey of people aged 65 years and over.

* Corresponding author: Dr P. J. Moynihan, fax. 0191 2225928, email p.j.moynihan@ncl.ac.uk 
men ${ }^{(11)}$. The main food and drink contributors were sweetened grains (cookies, cakes), sugars, sweets (table sugar, honey, syrups, candies, jams, jellies, gelatine desserts), soft drinks (colas, ginger ale, root beer), and milk/milk products (chocolate milk, ice cream, sweetened yoghurt) ${ }^{(12)}$. Intake was highest in the lowest income group ${ }^{(11)}$. In the free-living sample of the UK National Diet and Nutrition Survey of people aged 65 years and over (NDNS), NMES intake was $11.3 \%$ of food energy $(44 \mathrm{~g} / \mathrm{d})$ for women and $12.6 \%$ of food energy $(64 \mathrm{~g} / \mathrm{d})$ for men; $58 \%$ of NDNS participants had an intake of $\geq 11 \%$ food energy ${ }^{(13)}$. The main food and drink sources of NMES were sugar, preserves, sweet spreads and confectionery, providing $42 \%$ of total intake, of which table sugar provided $28 \%$ and preserves and sweet spreads $10 \%$. Cereals and cereal products provided $29 \%$, mostly from buns, cakes and pastries $(13 \%)$. Soft drinks, including fruit juices, provided a further $13 \%{ }^{(13)}$. However, these data are now a decade old.

It is necessary to have accurate and up-to-date information on the food and drink sources and frequency of intake of NMES in older adults to design effective interventions. The aims of this paper were therefore to report on sugars intake (amount and frequency) and the percentage contribution of different food groups to intake of sugars by older adults from socially deprived backgrounds.

\section{Methods}

\section{Study design}

This paper reports results from the baseline evaluation (August 2002 to February 2003) of a cluster- randomised community-based peer-led intervention with older adults living in sheltered housing schemes in relatively socially deprived areas of North East England ${ }^{(14)}$. Ethical approval was obtained from the Local Research Ethics Committees in the study areas; the nature of the study was described verbally and written consent was obtained from all participants.

\section{Participants}

Townsend scores ${ }^{(15)}$ were computed from the postal codes of sheltered housing schemes as a measure of social and economic deprivation. A nutritionist visited schemes in the most deprived areas to recruit study participants aged $65-85$ years who lived alone, with a partner or one other person. Exclusion criteria included: on a therapeutic diet; member of minority ethnic group; living with extended family; living in an institution; severe visual or hearing impairment; planning to move accommodation. Recruitment ceased when thirty-two schemes with nine participants per scheme had been recruited.

\section{Diet assessment}

Two 3-d estimated food diaries ( 2 weekdays and 1 weekend day), collected on the fourth day with an interview and separated by 2 weeks, were used to assess usual dietary intake. A photographic food atlas was used to ascertain portion sizes ${ }^{(16)}$. Dietary data were coded using an electronic version of the most up to date UK McCance and Widdowson food composition tables, entered into a purpose-designed Microsoft Access database (Microsoft Access 2000; version 9) and nutrient intakes calculated.

\section{Calculation of NMES and sources of NMES}

NMES are chemically indistinguishable from other sugars, therefore NMES content of foods and drinks must be estimated from total sugar content ${ }^{(17)}$. The method used in this study was that developed by the Human Nutrition Research Centre (HNRC) at Newcastle University ${ }^{(2,18)}$ : NMES are defined as 'added sugars' plus sugars from fruit in fruit juices and other soft drinks. Fruit sugars deriving from the fruit in jams and yoghurts, and from dried fruit, are classified as intrinsic, whilst all sugars in chocolate are classified as NMES ${ }^{(2,18)}$. Foods were grouped to determine the relative importance of dietary sources of NMES, based on food groups developed by the HNRC ${ }^{(18)}$.

\section{Calculation of frequency of intake of NMES}

Because participants recorded in their food diaries the time of consumption of all foods and drinks it was possible to identify separate eating occasions. All eating occasions on which NMES-containing foods or drinks were consumed were identified. Even if more than one NMES-containing food or drink item was consumed at an eating occasion, this was counted as one. The number of eating occasions on which NMES were consumed was summed for each day and an average of $6 \mathrm{~d}$ calculated.

\section{Statistical analysis}

The mean of 6-d dietary intake was calculated for each participant in Access and imported into SPSSb version 12.0.1 (Statistical Package for Social Science Inc., Chicago, IL, USA) for analysis. Descriptive data are presented as means and standard deviations. Differences in NMES intake between groups meeting and not meeting the WHO recommendation for frequency of intake ( $\leq 4$ times/d) were compared using $t$ tests.

\section{Results}

Of 304 residents who volunteered to participate, 201 (170 women, thirty-one men) completed both 3-d food diaries. Mean Townsend Score was 4.79 (SD 1.89), mean age was 76.7 (SD 5.5) years (women 76.8 (SD 5.7) years; men 76.0 (SD 4.2) years), and mean BMI was 29.2 (SD 5.3) $\mathrm{kg} / \mathrm{m}^{2}$ (women 29.6 (SD 5.4) kg/m²); men 27.4 (SD 4.1) $\mathrm{kg} / \mathrm{m}^{2}$ ). Mean total energy intake was 5.96 (SD 1.44) MJ/d for women and 8.42 (SD 2.02) MJ/d for men. The mean physical activity level (PAL; ratio of energy intake to BMR) was 1.2 , and $44.8 \%$ of participants had a PAL $<1.1$.

Total sugars intake represented 19.6 (SD 6.6) \%) of energy intake (76.8 (SD 34.5) g/d), NMES 9.3 (SD 4.9) \% of energy intake (37.1 (SD 23.7) g/d), and intrinsic and milk sugars 10.3 (SD 4.7) \% of energy intake (39.7 (SD 19.6) g/d). Eighty-one participants $(40 \cdot 3 \%)$ exceeded the WHO recommendation of $\leq 10 \%$ energy from NMES.

Mean daily frequency of NMES intake was 3.4 (SD 1.2), and ranged from 0.5 to 8.5 , with fifty-three participants $(26.4 \%)$ exceeding the recommendation of $\leq 4$ times/d. Participants who met the recommendation obtained 8.0 (SD 4.1) \% energy from NMES compared with 12.9 (SD 5.0) $\%$ for those with an intake $>4$ times/d $(P<0 \cdot 0005)$. 
The relative contribution of food groups to total sugars, intrinsic and milk sugars, and NMES is detailed in Table 1. The food groups that made the greatest contribution to NMES intake were biscuits and cakes, soft drinks (particularly fruit juices), table sugar, confectionery, and sweet puddings. Within the soft drinks group, fruit juice was the largest contributor to NMES. Other sources within this group were ginger ale as a mixer, tonic water and lemonade as an alternative to alcoholic drinks, fruit flavoured squash and fruit juice drinks. The group 'other sources' made a substantial contribution to total sugars and intrinsic and milk sugars, with the greatest contribution from vegetables and potatoes, white tea and coffee, cereals (e.g. breads, rice, pasta), and milky drinks (e.g. Ovaltine). The defined food groups accounted for all but $5 \cdot 1 \mathrm{~g}$ of NMES, which mostly came from milky drinks, soup, alcohol, and vegetables and potatoes.

\section{Discussion}

In this sample of older adults from socially deprived backgrounds, NMES accounted for $9.3 \%$ of energy intake and mean intake was $37 \mathrm{~g} / \mathrm{d}$, both less than the maximum recommended values of $\leq 10 \%$ energy or $60 \mathrm{~g} / \mathrm{d}^{(3,4)}$. These values are somewhat lower than those reported for the NDNS of older adults ${ }^{(13)}$. The NDNS and HNRC methods for calculating NMES produce only minor differences in estimated intake and methodological issues are unlikely to account for these differences ${ }^{(2)}$. Although mean NMES intake was below the maximum recommended intake, $40.3 \%$ of participants had an intake $>10 \%$ energy, compared with the NDNS value of $58 \%^{(13)}$.

The food groups that made the greatest contribution to NMES intakes (biscuits and cakes (18.9\%), soft drinks $(13.1 \%)$ and table sugar $(11.1 \%))$, were similar to findings from the $\mathrm{NDNS}^{(13)}$ and the CSFII ${ }^{(12)}$. This compares with
English adolescents, for whom the greatest contribution came from soft drinks (38\%), confectionery $(23 \%)$ and biscuits and cakes $(15 \%)^{(19)}$. NMES also made a greater contribution to energy intake $(16 \cdot 1 \%)^{(19)}$. The identification of the main sources of NMES in the diets of older adults should help health educators to provide more specific dietary advice.

Dental caries is associated with both NMES intake and with frequency of consumption ${ }^{(7)}$. Further analysis of the NDNS showed that more frequent intake of foods and drinks high in sugars was associated with a higher prevalence of root caries, with a sharp increase at $\geq 9$ times/ $\mathrm{d}^{(10)}$. The mean frequency of intake found in this study ( 3.4 times/d) meets the WHO and Eurodiet recommendation of $\leq 4$ times $/ \mathrm{d}^{(3,6)}$. However, a quarter of participants exceeded this target, and they had significantly higher NMES intakes compared with those who met the frequency recommendation, illustrating how quantity and frequency are related ${ }^{(3,7)}$. Advice to reduce the frequency of consumption of NMES is likely also to reduce quantity.

The relatively low proportion of men who participated in this study $(15.4 \%)$ is a limitation: the small number of other studies that include information on NMES intakes suggest that men obtain a higher percentage of energy from NMES than do women ${ }^{(13,20,21)}$, although the main sources of NMES did not vary by gender in the NDNS ${ }^{(13)}$ or CSFII ${ }^{(12)}$. The average PAL of the group was low with a high percentage of participants with an energy intake:BMR $<1 \cdot 1$. The mean PAL was, however, comparable to that reported in the $\mathrm{NDNS}^{(13)}$. The relatively low PAL reflects the high body weight and low energy intakes. Since the Schofield equation applies to all those over 60 years, it does not take into account decreasing lean mass with increasing age and so becomes less applicable as age increases. 'Dieting' and under-reporting by some cannot be ruled out and the latter may occur as a result of memory lapses, lack of interest or socially desirable reporting.

Table 1. Contribution of food groups to intake of total sugars, non-milk extrinsic sugars (NMES) and intrinsic and milk sugars (g/d) and as a percentage of intake

(Mean values with standard deviations)

\begin{tabular}{|c|c|c|c|c|c|c|c|c|c|c|c|c|}
\hline & \multicolumn{4}{|c|}{ Total sugars } & \multicolumn{4}{|c|}{ NMES } & \multicolumn{4}{|c|}{ Intrinsic and milk sugars } \\
\hline & \multicolumn{2}{|c|}{$g / d$} & \multicolumn{2}{|c|}{$\%$} & \multicolumn{2}{|c|}{$g / d$} & \multicolumn{2}{|c|}{$\%$} & \multicolumn{2}{|c|}{$g / d$} & \multicolumn{2}{|c|}{$\%$} \\
\hline & Mean & SD & Mean & SD & Mean & SD & Mean & SD & Mean & SD & Mean & SD \\
\hline Confectionery & 4.5 & $6 \cdot 6$ & 5.5 & $7 \cdot 1$ & 3.9 & $6 \cdot 2$ & $9 \cdot 7$ & $12 \cdot 6$ & 0.6 & 1.3 & 1.3 & 3.3 \\
\hline Sweets and chocolates & 3.3 & $6 \cdot 2$ & 3.9 & 6.8 & $3 \cdot 2$ & $6 \cdot 0$ & $7 \cdot 4$ & $12 \cdot 1$ & 0.1 & 0.5 & 0.2 & $2 \cdot 1$ \\
\hline Ice cream & $1 \cdot 2$ & $2 \cdot 1$ & 1.5 & $2 \cdot 7$ & 0.7 & 1.4 & 2.4 & $5 \cdot 1$ & 0.5 & 1.2 & 1.1 & $2 \cdot 4$ \\
\hline Soft drinks & 6.0 & 11.5 & 6.5 & 9.6 & $6 \cdot 0$ & 11.5 & $13 \cdot 1$ & $17 \cdot 9$ & 0.0 & 0.4 & 0.1 & 1.3 \\
\hline Soft drinks & 1.8 & 4.3 & $2 \cdot 3$ & 4.6 & 1.7 & $4 \cdot 2$ & 4.8 & $9 \cdot 6$ & 0.0 & 0.4 & 0.1 & 1.3 \\
\hline Fruit juice & 4.2 & $10 \cdot 3$ & 4.2 & 8.4 & $4 \cdot 2$ & $10 \cdot 3$ & 8.4 & $15 \cdot 8$ & 0.0 & 0.0 & 0.0 & 0.0 \\
\hline Biscuits and cakes & $7 \cdot 0$ & 6.8 & 9.8 & 9.3 & $6 \cdot 0$ & 5.7 & $18 \cdot 9$ & 17.5 & 1.0 & $2 \cdot 1$ & $2 \cdot 7$ & $5 \cdot 3$ \\
\hline Table sugar & $5 \cdot 7$ & 11.4 & 6.4 & 11.9 & $5 \cdot 7$ & 11.4 & $11 \cdot 1$ & $19 \cdot 6$ & 0.0 & 0.0 & 0.0 & 0.0 \\
\hline Dairy, milk and yoghurt & 8.6 & $7 \cdot 2$ & 11.5 & 9.2 & 1.3 & $2 \cdot 7$ & $4 \cdot 3$ & 10.9 & $7 \cdot 2$ & 5.5 & $18 \cdot 6$ & $12 \cdot 6$ \\
\hline Sweet puddings & 4.6 & 5.8 & $6 \cdot 4$ & $7 \cdot 7$ & $3 \cdot 1$ & 4.1 & $9 \cdot 1$ & 11.4 & 1.6 & $2 \cdot 1$ & 4.5 & $6 \cdot 3$ \\
\hline Breakfast cereals & $3 \cdot 1$ & 4.4 & 4.0 & 4.8 & $2 \cdot 4$ & 3.7 & 7.9 & 10.4 & 0.7 & $1 \cdot 7$ & 1.8 & 4.6 \\
\hline Fruit & $16 \cdot 1$ & $15 \cdot 9$ & $19 \cdot 3$ & 14.5 & 0.9 & $2 \cdot 2$ & $2 \cdot 6$ & $6 \cdot 0$ & $15 \cdot 2$ & $15 \cdot 6$ & 31.8 & $20 \cdot 1$ \\
\hline Fresh fruit & $12 \cdot 9$ & $12 \cdot 4$ & $15 \cdot 9$ & $13 \cdot 3$ & $0 \cdot 1$ & 0.5 & 0.2 & $2 \cdot 4$ & $12 \cdot 9$ & $12 \cdot 4$ & $27 \cdot 4$ & $19 \cdot 3$ \\
\hline Dried fruit & 1.6 & 6.7 & 1.4 & 4.4 & 0.0 & 0.0 & 0.0 & 0.0 & 1.6 & $6 \cdot 7$ & $2 \cdot 3$ & $7 \cdot 0$ \\
\hline Canned/stewed fruit & 1.6 & 3.3 & 2.0 & 4.0 & 0.8 & $2 \cdot 2$ & $2 \cdot 3$ & 5.5 & 0.8 & 1.5 & $2 \cdot 1$ & 4.2 \\
\hline Preserves and syrups & 3.5 & $5 \cdot 3$ & 4.4 & 6.8 & $2 \cdot 7$ & 4.7 & $7 \cdot 1$ & 11.7 & 0.8 & $1 \cdot 1$ & $2 \cdot 1$ & 3.4 \\
\hline Other sources & $17 \cdot 7$ & $7 \cdot 1$ & $26 \cdot 2$ & $12 \cdot 8$ & $5 \cdot 1$ & 5.6 & $16 \cdot 2$ & $17 \cdot 4$ & $12 \cdot 6$ & $5 \cdot 0$ & $37 \cdot 1$ & $18 \cdot 7$ \\
\hline Total & $76 \cdot 8$ & 34.5 & 100 & & 37.1 & $23 \cdot 7$ & 100 & & 39.7 & $19 \cdot 6$ & 100 & \\
\hline
\end{tabular}


The reported intake of NMES may therefore be an underestimation.

Despite having an on-site warden, residents living in sheltered accommodation did so relatively independently. Participants had their own kitchens and were responsible for providing their own meals, although some had assistance with shopping. As such, they could be considered as largely representative of the vast majority of older adults living independently ${ }^{(22)}$.

Greater retention of teeth presents its own challenges with the increased prevalence and susceptibility to dental caries. It is important, therefore, that older adults also appreciate the necessity of reducing both quantity and frequency of intake of NMES. The findings from this study suggest that they should be advised to reduce intake of biscuits and cakes, table sugar, confectionery, and sweet puddings, and to consume fruit juice with meals, rather than between, to reduce frequency of intake.

\section{Acknowledgements}

Data used in the analysis within this paper was collected as part of a wider peer-led project commissioned by the Food Standards Agency. The views expressed are those of the authors and not necessarily those of the Food Standards Agency. There are no conflicts of interest. J. Bradbury was the lead author, C. E. Mulvaney collected the data, A. J. Adamson, J. C. Mathers and C. J. Seal were members of the project advisory group and advised on drafts of the manuscript, and P. J. Moynihan was the project Principal Investigator.

\section{References}

1. Department of Health (1989) Dietary Sugars and Human Disease. Report on Health and Social Subjects 37. London: HMSO.

2. Kelly SAM, Summerbell C, Rugg-Gunn AJ, Adamson A, Fletcher E \& Moynihan PJ (2005) Comparison of methods to estimate non-milk extrinsic sugars and their application to sugars in the diet of young adolescents. Br J Nutr 94, 114-124.

3. World Health Organization (2003) Diet, Nutrition and the Prevention of Chronic Diseases: Report of a Joint WHO/FAO expert consultation. WHO Technical Report Series, 916. Geneva: World Health Organization.

4. Department of Health (1991) Dietary Reference Values for Food Energy and Nutrients for the United Kingdom. Report on Health and Social Subjects No. 41. London: HMSO.

5. Sheiham A (2001) Dietary effects on dental diseases. Public Health Nutr 4, 569-591.
6. Eurodiet Core Report (2001) Nutrition and diet for healthy lifestyles in Europe: science and policy implications. Public Health Nutr 4, 265-273.

7. Moynihan P \& Petersen PE (2004) Diet, nutrition and the prevention of dental diseases. Public Health Nutr 7, 201-226.

8. Steele JG, Sheiham A, Marcenes W \& Walls AWG (1998) National Diet and Nutrition Survey: people aged 65 years and over. Volume 2: Report of the oral health survey. London: The Stationery Office.

9. Strategy Review Group (2005) Oral health and older people. Gerodontology 22, Suppl. 1, 12-15.

10. Steele JG, Sheiham A, Marcenes W, Fay N \& Walls AWG (2001) Clinical and behavioural risk indicators for root caries in older people. Gerodontology 18, 95-101.

11. Krebs-Smith SM (2001) Choose beverages and foods to moderate your intake of sugars: measurement requires quantification. J Nutr 131, 527S-535S

12. Guthrie JF \& Morton JF (2000) Food sources of added sweeteners in the diets of Americans. $J$ Am Diet Assoc 100, 43-48, 51.

13. Finch S, Doyle W, Lowe C, Bates C, Prentice A, Smithers G \& Clarke PC (1998) National Diet and Nutrition Survey: people aged 65 years and over. Volume 1: Report of the Diet and Nutrition Survey. London: The Stationery Office.

14. Moynihan P, Zohoori V, Seal C, Hyland R \& Wood C (2006) Design and Evaluation of Peer-led Community Based Food Clubs: a Means to Improve the Diets of Older People from Socially Deprived Backgrounds. London: Food Standards Agency.

15. Townsend P, Phillimore P \& Beattie A (1988) Health and Deprivation: Inequality and the North. London: Routledge.

16. Nelson M, Atkinson M \& Meyer J (1997) Food Portion Sizes: A Photographic Atlas. London: MAFF Publications.

17. Kelly SAM, Moynihan PJ, Rugg-Gunn AJ \& Summerbell CD (2003) Review of methods used to estimate non-milk extrinsic sugars. J Hum Nutr Diet 16, 27-38.

18. Rugg-Gunn AJ, Adamson AJ, Appleton DR, Butler TJ \& Hackett AF (1993) Sugars consumption by 379 11-12 year-old English children in 1990 compared with results in 1980. J Hum Nutr Diet 6, 419-431.

19. Rugg-Gunn AJ, Fletcher ES, Matthews JNS, Hackett AF, Moynihan PJ, Kelly SAM, Adams J, Mathers JC \& Adamson AJ (2007) Changes in consumption of sugars by English adolescents. Public Health Nutr 10, 354-363.

20. Swan G (2004) Findings from the latest National Diet and Nutrition Survey. Proc Nutr Soc 63, 505-512.

21. Bolton-Smith C \& Woodward M (1995) Intrinsic, non-milk extrinsic and milk sugar consumption by Scottish adults. J Hum Nutr Diet 8, 35-49.

22. Tomassini C (2005) Family and living arrangements. In Focus on Older People, pp. 11-20 [A Soule, P Babb, M Evandrou, $\mathrm{S}$ Balchin and L Zealey, editors]. Office for National Statistics: http://www.statistics.gov.uk/downloads/theme_compendia/foop05/Olderpeople2005.pdf. 